\title{
On the Basic States of One-Dimensional Disordered Structures
}

\author{
L. N. Grenkova, S. A. Molčanov, and Ju. N. Sudarev \\ Department of Mathematics, Moscow State University, Moscow V-234, USSR
}

Abstract. The purpose of this paper is to study a limit probability distribution of the set of the first $\kappa$ eigenvalues $\lambda_{1}(\mathscr{L})<\lambda_{2}(\mathscr{L})<\ldots<\lambda_{\kappa}(\mathscr{L})$ (with a fixed $\kappa$ and $\mathscr{L} \rightarrow \infty)$ of the boundary problem on the interval $[0, \mathscr{L}]$

$$
\begin{gathered}
H y=-\frac{d}{d t} a(t, \omega) \frac{d y}{d t}+q(t, \omega) y=\lambda y, \\
y(0)=y(\mathscr{L})=0,
\end{gathered}
$$

where $a(t, \omega), q(t, \omega)$ are the random stationary processes. Particularly the question of the repulsion between the first eigenvalues (small energetic levels) is studied. It has been proved that in the "divergent" case $(q(t, \omega)=0, a(t, \omega) \neq 0)$ levels repulsion exists. As for the "potential" case $(a(t, \omega) \equiv 1, q(t, \omega) \neq 0)$ there is not any repulsion at all. This is one of the main differences between these two cases.

\section{Introduction}

Let $H(\omega)=-\frac{d}{d t} a(t, \omega) \frac{d}{d t}+q(t, \omega)$ be a one-dimensional random Schrödinger operator with stationary coefficients $a(t, \omega)>0, q(t, \omega)$ (on a probability space $(\Omega, \mathscr{F}, P)$ ), describing the quantum-mechanical behaviour of an electron in a random medium. As for its spectral properties, it is possible to study them either in $\mathscr{L}^{2}\left(R^{1}\right)$ or in $\mathscr{L}^{2}(-\mathscr{L}, \mathscr{L})$ when $\mathscr{L} \rightarrow \infty$. The latter is more important for physical applications. A restriction $H_{\mathscr{L}}(\omega)$ of the operator $H(\omega)$ on $\mathscr{L}^{2}(\mathscr{L}, \mathscr{L})$ is determined by some classical boundary conditions $\gamma$. Since the coefficients $a(t, \omega), q(t, \omega)$ are stationary, many properties of the operator $H(\omega)$ on a large segment are independent of the segment's position. That is why we shall further consider $[0, \mathscr{L}]$ instead of $[-\mathscr{L}, \mathscr{L}]$ since it is technically simpler. We shall study asymptotic of the eigenvalues (the energetic levels) $\lambda_{i}(\mathscr{L}, \omega)$ when $\mathscr{L} \rightarrow+\infty$ and the eigenfunctions for the following boundary problem:

$$
H \psi(t)=\lambda \psi(t) ; \quad \gamma(\psi(t))=0, \quad t=0, \mathscr{L} .
$$


If $N_{\mathscr{L}}(\lambda)=\sum_{\lambda_{i}(\mathscr{L}) \leqq \lambda} 1$, then under very general conditions there exists a nonrandom continuous limit

$$
N(\lambda)=\lim _{\mathscr{L} \rightarrow+\infty} \frac{1}{\mathscr{L}} N_{\mathscr{L}}(\lambda), \text { where } \mathscr{L} \rightarrow+\infty,
$$

which is called the limit spectral distribution function [I].

If $q(t, \omega) \geqq$ const with probability 1 (i.e. $P$-almost surely) then there exists a point $\bar{\lambda}$ such that $N(\bar{\lambda})=0$ and $N(\lambda)>0$ when $\lambda>\bar{\lambda}$. The point $\bar{\lambda}$ is $(P$-almost surely) the left end of the spectrum $S(H(\omega))$ of $H(\omega)$ in $\mathscr{L}^{2}\left(R^{1}\right)$.

The character decay of $N(\lambda)$ at the left end of the spectrum in various models is different. Particularly in a model studied in [2] when $q \equiv 0$ ("divergent case") the following asymptotics for $N(\lambda)$ was found

$$
N(\lambda) \sim \text { const } \cdot \lambda^{1 / 2}, \text { when } \lambda \rightarrow \bar{\lambda}=0 .
$$

However in a model with $a \equiv 1$ and $q(t, \omega)=\sum_{i} \delta\left(t-\tau_{i}\right)$, where $\tau_{i}$ are points of a Poisson flow (this potential is often used in physics and is called $\delta$-potential) the asymptotics for $N(\lambda)$ is a different one. Namely, using the results of [3] one can obtain

$$
N(\lambda) \sim \text { const } \cdot \exp \left(-\pi / \lambda^{1 / 2}\right) \quad \text { when } \quad \lambda \rightarrow \bar{\lambda}=0 .
$$

The purpose of this work is to study a limit probability distribution of the set of the first $k$ eigenvalues $\lambda_{1}, \lambda_{2} \ldots \lambda_{k}$ with a fixed $k$ and $\mathscr{L} \rightarrow+\infty$ and to find out a structure for the corresponding eigenfunctions. A similar problem was solved in $[4,5]$ for eigenvalues in a neighborhood of a fixed internal point of the spectrum. Namely, for the case $a(t, \omega) \equiv 1$ and $q(t, \omega)=F\left(x_{t}(\omega)\right)$, where $x_{t}(\omega)$ is a diffusion process on a smooth compact manifold $K$ and $F: K \rightarrow R^{1}$ is a smooth Morse function such that $\min _{K} F=0$, it was proved that when $\lambda_{0}>0$ and $a, b(a<b)$ are fixed, a point process $\left\{\lambda_{i}(\mathscr{L})\right\}$ is asymptotically a Poisson process $\left(\right.$ in scale $\left.\frac{1}{\mathscr{L}}\right)$ in the neighbourhood $\Delta_{a, b}=\left(\lambda_{0}-a / \mathscr{L}, \lambda_{0}+b / \mathscr{L}\right)$ with the parameter $n\left(\lambda_{0}\right)$, where $n\left(\lambda_{0}\right)=\frac{d N\left(\lambda_{0}\right)}{d \lambda}$ is a state density. Its continuity was also eastablished. The eigenfunctions $\psi_{\mathscr{L}}(t, \mathscr{L})$ corresponding to the spectral interval $\Delta_{a, b}$ are decreasing exponentially. That is for any function $\psi_{i}(t, \mathscr{L}, \omega): \lambda_{i}(\mathscr{L}) \in \Delta_{a, b}$ there exists a point $\tau_{i} \in[0, \mathscr{L}]$ such that

$$
\left(\left|\psi_{i}\left(t-\tau_{i}\right)\right|^{2}+\left|\frac{d}{d t} \psi_{i}\left(t-\tau_{i}\right)\right|^{2}\right)^{1 / 2} \leqq c(\varepsilon, \omega) \exp \left(\left(-\alpha\left(\lambda_{0}\right)+\varepsilon\right)\left|t-\tau_{i}\right|\right)
$$

Here $\varepsilon>0$ is a fixed small constant, $\alpha\left(\lambda_{0}\right)$ is a nonrandom positive function (Liapunov index) and $c(\varepsilon, \omega)$ is a constant bounded in probability when $\mathscr{L} \rightarrow+\infty$. Note that the asymptotic Poisson property of the spectrum $\left\{\lambda_{i}(\mathscr{L})\right\}$ near the point $\lambda_{0}>0$ is interpreted physically as the absence of any interaction between the spectral levels.

The results obtained in [5] alter the viewpoint suggested earlier, according to which there exists a repulsion between spectral levels in quantum disordered 
systems (see the discussion in [5]). After [5] had been published it was suggested in a number of new papers in physics that the levels repulsion hypothesis might be secured at least for small energies $\lambda=o(1)$ when $\mathscr{L} \rightarrow+\infty$.

This hypothesis turns out to be true for some cases but for others it turns out to be false even for small energies. To distinguish these cases a supplementary classification of hamiltonians is necessary. In particular the spectral properties of a "divergent" one-dimensional hamiltonian $H=-\frac{d}{d t} a(t, \omega) \frac{d}{d t}$ at the end of the spectrum are similar to those of the operator $H_{0}=-\frac{d^{2}}{d t^{2}}$. Namely there is a strong repulsion between the small levels and the corresponding eigenfunctions are not localized.

Similar effects were observed of the spectrum end structure for difference analogues of divergent operators. Moreover, this was true even for the multi-dimensional case $[12,13]$. Apparently, it is possible to prove that the situation for a "potential operator" $H(\omega)=-d^{2} / d t^{2}+\sum_{i} \xi_{i} \delta\left(t-\tau_{i}\right)$ with a generalized $\delta$-potential at the end of the spectrum is in general similar to that near the point $\lambda_{0}>0$. Now we can formulate more precisely our results.

The first one in physical terms is the following: let us consider a divergent operator $H=-\frac{d}{d t} a(t, \omega) \frac{d}{d t}$ on $[0, \mathscr{L}]$. Let $a(t, \omega)$ be a stationary random process with an finite expectation $\mathrm{M}\left(\frac{1}{a(t, \omega)}\right)=\alpha$ and $\mathrm{M}\left(\frac{1}{a^{2}(t, \omega)}\right)<\infty$. Let the process $\left(\frac{1}{a\left(\frac{t}{\varepsilon}, \omega\right)}-\alpha\right) / \varepsilon^{1 / 2}$ weakly converge to Gaussian white noise; then

$$
\lambda_{i+1}(\mathscr{L}, \omega)-\lambda_{i}(\mathscr{L}, \omega)=\lambda_{i+1}^{0}(\mathscr{L})-\lambda_{i}^{0}(\mathscr{L})+\frac{\xi_{i}(\mathscr{L}, \omega)}{\mathscr{L}^{5 / 2}}
$$

where $\lambda_{i}^{0}(\mathscr{L})$ are the eigenvalues of the averaged operator

$$
H_{0}=-\frac{1}{M\left(\frac{1}{a(t, \omega)}\right)} \frac{d^{2}}{d t^{2}}
$$

and $\xi_{i}(\mathscr{L}, \omega), i=1,2 \ldots k$ have joint Gaussian distribution when $k$ is fixed and $\mathscr{L} \rightarrow+\infty$.

The second result deals with the "potential case." Let $H=-\frac{d^{2}}{d t^{2}}+\sum_{i} \xi_{i} \delta\left(t-\tau_{i}\right)$, where $\tau_{i}$ are the points of Poisson flow, $\tau_{i}$ do not depend on $\xi_{i}$ and $\xi_{i}$ are independent random variables having the same exponential distribution; then

$$
\lambda_{2}-\lambda_{1}, \lambda_{3}-\lambda_{2}, \ldots, \lambda_{k}-\lambda_{k-1}
$$

are asymptotically independent and after corresponding normalization they have a limit exponential distribution. 
In the divergent case a Liapunov index is degenerated, $\alpha(0)=0$, while in the potential case $\alpha(0)>0$, which accounts for the difference between both cases. (The definition of $\alpha(\lambda)$ see in $[1,4]$ ).

Let us proceed to the exact formulations starting with the divergent case.

\section{Divergent Case}

Let $H(\omega)=-\frac{d}{d t} a(t, \omega) \frac{d}{d t}$ be an one-dimensional random operator on $[0,+\infty)$, where $a(t, \omega)$ is a stationary process satisfying the following supplementary conditions:

1. $0<\alpha=\mathrm{M}\left(\frac{1}{a(t, \omega)}\right)<\infty ; \quad \mathrm{D}\left(\frac{1}{a(t, \omega)}\right)<\infty$.

2. The distribution of the normalized process

$$
\mathscr{L}^{1 / 2}\left[\int_{0}^{t} \frac{d s}{a(s \mathscr{L}, \omega)}-t \alpha\right]=\xi_{\mathscr{L}}(t), \quad t \in[0,1]
$$

weakly converges when $\mathscr{L} \rightarrow+\infty$ to the distribution of the Wiener process with some variance $\sigma^{2}>0$. Thus in the sense of closeness of distributions in Levy-Prohorov metric

$$
\mathscr{L}^{1 / 2}\left(\int_{0}^{t} \frac{d s}{a(s \mathscr{L}, \omega)}-t \alpha\right) \sim \sigma w_{t}, \quad t \in[0,1], \mathscr{L} \rightarrow+\infty .
$$

Condition 2 is valid at least for a large class of stationary Markov processes. We shall further give some examples where conditions 1, 2 are valid and the variance of the limit Wiener process can be calculated. Let us consider the spectral problem

$$
H y=-\frac{d}{d t} a(t, \omega) \frac{d}{d t} y=\lambda y, t \in[0, \mathscr{L}], y(0)=y^{\prime}(\mathscr{L})=0
$$

Note that a choice of such boundary conditions is connected with considerable simplification of some formulae; however it is not difficult to study the general case

$$
y(0)+h_{1} y^{\prime}(0)=0, y(\mathscr{L})+h_{2} y^{\prime}(\mathscr{L})=0 .
$$

Theorem I. The set of the first $k$ eigenvalues of the spectral problem (I.1) when $\mathscr{L} \rightarrow+\infty$ and $k>0$ is fixed can be represented in the form

$$
\begin{aligned}
\lambda_{i}(\mathscr{L}, \omega) & =\frac{\pi^{2}(1+2 i)^{2}}{4 \mathscr{L}^{2} \alpha}\left(1+\frac{\xi_{i}(\mathscr{L}, \omega)}{\mathscr{L}^{1 / 2}}\right), \\
\alpha & =\mathrm{M}\left(\frac{1}{a(t, \omega)}\right) ;
\end{aligned}
$$


where $\xi_{i}(\mathscr{L}, \omega), 0 \leqq i \leqq k-1$ is a random vector having a limit Gaussian distribution with zero mathematical expectation and covariance matrix $\sigma^{2} B=\sigma^{2}\left(b_{i j}\right)$, where $b_{i j}$ are constants that can be effectively calculated ${ }^{1}$.

Proof. The idea of the proof is different from that of [2] but has many points in common with [12] which, however, deals with discrete time. We substitute variables putting $x=t / \mathscr{L}$, then our problem becomes equivalent to the following one

$$
H y=-\frac{d}{d x} a(x \mathscr{L}, \omega) \frac{d}{d x} y=\lambda \mathscr{L}^{2} y, x \in[0,1] ; y(0)=y^{\prime}(1)=0 .
$$

Let us denote the eigenvalues of the boundary problem (I.4) by $\mu_{i}(\mathscr{L}), i=0,1,2 \ldots$. Then $\mu_{i}(\mathscr{L})=\lambda_{i}(\mathscr{L}) \cdot \mathscr{L}^{2}$, where $\lambda_{i}(\mathscr{L}), i=0,1,2 \ldots$ are eigenvalues of the boundary problem (I.1).

Now let us consider a homogeneous equation

$$
H y=0 .
$$

This equation has two independent solutions

$$
y_{1}(x)=\text { const } ; y_{2}(x)=\int_{0}^{x} \frac{d u}{a(u \mathscr{L}, \omega)} .
$$

The Green function of a nonhomogeneous problem

$$
\begin{aligned}
& H y=f, \\
& y(0)=y^{\prime}(1)=0
\end{aligned}
$$

can be obtained with the help of these solutions in a standard way.

Now the given spectral problem is reduced to an integral equation

$$
\begin{aligned}
y(x) & =\int_{0}^{1} G(x, z, \omega) \lambda \mathscr{L}^{2} y(z) d z \\
& =\lambda \mathscr{L}^{2} \int_{0}^{x} y(z) d z \int_{0}^{z} \frac{d u}{a(\mathscr{L} u, \omega)}+\lambda \mathscr{L}^{2} \int_{0}^{x} \frac{d u}{a(\mathscr{L} u, \omega)} \int_{x}^{1} y(z) d z .
\end{aligned}
$$

Further we shall use the fact that

$$
\mathscr{L}^{1 / 2}\left(\int_{0}^{t} \frac{d s}{a(s \mathscr{L}, \omega)}-t \alpha\right)=\xi_{\mathscr{L}}(t) \rightarrow \sigma w_{t} \quad \text { when } \quad \mathscr{L} \rightarrow+\infty
$$

under condition 2 in the sense of distribution, where $\alpha=\mathrm{M}\left(\frac{1}{a(s, \omega)}\right), w_{t}$ is a Wiener process. So we substitute $\left(z \alpha+\xi_{\mathscr{L}}(z) / \mathscr{L}^{1 / 2}\right)$ and $\left(x \alpha+\xi_{\mathscr{L}}(x) / \mathscr{L}^{1 / 2}\right)$ for $\int_{0}^{z} \frac{d u}{a(\mathscr{L} u, \omega)}$ and $\int_{0}^{x} \frac{d u}{a(\mathscr{L} u, \omega)}$ in (I.7) respectively, and obtain

$1 \quad b_{i j} \neq 0, i \neq j$ so that the $\lambda_{i}$ in general are asymptotically dependent even after normalization 


$$
y(x)=\int_{0}^{1}\left(G_{0}(x, z)+\frac{1}{\mathscr{L}^{1 / 2}} G_{1}^{(\mathscr{L})}(x, z, \omega)\right) \lambda \mathscr{L}^{2} y(z) d z
$$

Thus the Green function of the boundary problem (1.6) is presented in the form of the sum of the Green function $G_{0}(x, z)$ of the nonrandom boundary problem

$$
H_{0} y=-\frac{1}{\alpha} \frac{d^{2}}{d x^{2}} y=\lambda \mathscr{L}^{2} y, \quad y(0)=y^{\prime}(1)=0
$$

and a certain asymptotically Gaussian and infinitely small kernel $\frac{1}{\mathscr{L}^{1 / 2}} G_{1}^{(\mathscr{L})}(x, z, \omega)$, which is evidently expressed asymptotically through a Wiener process.

Now we shall use classical perturbation theory. Let $\mu_{0, i}=\lambda_{0, i} \mathscr{L}^{2}$ be the $i$-th eigenvalue of the nonrandom boundary problem (I.9), then

$$
y(x)=\mu_{0, i} \int_{0}^{1} G_{0}(x, z) y(z) d z=\mu_{0, i} \mathbb{G}_{0} y(x) .
$$

As the operator $\mathbb{G}_{0}$ is inverse to the iperator $H_{0} y=-\frac{1}{\alpha} \frac{d^{2}}{d x^{2}} y, y(0)=y^{\prime}(1)=0$, then it is easy to understand that

$$
\mu_{0, i}=\frac{1}{\alpha}\left(\frac{\pi}{2}+\pi i\right)^{2}=\frac{\pi^{2}}{4 \alpha}(1+2 i)^{2}, \quad i \geqq 0,
$$

and the normalized eigenfunction corresponding to the $i^{\text {th }}$ eigenvalue $\mu_{0, i}$ is

$$
y_{0, i}(x)=\sqrt{2} \sin \left(\left(\alpha \mu_{0, i}\right)^{1 / 2} x\right)=\sqrt{2} \sin \left(\beta_{i} x\right),
$$

where

$$
\beta_{i}=\frac{\pi(1+2 i)}{2} ; \quad \mu_{0, i}=\frac{\beta_{i}^{2}}{\alpha} ; \quad i \geqq 0 .
$$

The operator $\mathbb{G}_{1}$ connected with the kernel $G_{1}^{(\mathscr{L})}(x, z, \omega)$ is random, depends on $\mathscr{L}$ and has no limit when $\mathscr{L} \rightarrow \infty$ (there exists only a weak limit of its distribution). Therefore it is necessary to be careful using perturbation theory. Under condition 2 the operator $\mathbb{G}_{1}$ is bounded in probability: for any $\varepsilon>0$ there exists a constant $c=c(\varepsilon)>0$ such that

$$
P\left\{\left\|\mathbb{G}_{1}\right\|>c(\varepsilon)\right\}=P\left\{A_{\mathscr{L}}(\varepsilon)\right\} \leqq \varepsilon, \quad \varepsilon>0
$$

for all $\mathscr{L}>\mathscr{L}_{0}$.

Let a sufficiently large $\mathscr{L}$ and an elementary event $\omega$ be fixed. Consider the spectral problem (I.8):

$$
y=\mu\left(\mathbb{G}_{0}+\frac{\mathbb{G}_{1}}{\mathscr{L}^{1 / 2}}\right) y,
$$

where $\mu=\lambda \mathscr{L}^{2}$, 


$$
\begin{gathered}
\mathbb{G}_{0} y=\int_{0}^{1} G_{0}(x, z) y(z) d z, \\
\mathbb{G}_{1} y=\int_{0}^{1} G_{1}^{(\mathscr{L})}(x, z, \omega) y(z) d z .
\end{gathered}
$$

Following [14] we shall look for a solution $y_{i}$ corresponding to the $i^{\text {th }}$ eigenvalue $\mu_{i}$ of Eq. (I.11) in the form of a perturbation of the function $y_{0, i}$ choosing the normalization

$$
\left(y_{i}, y_{0, i}\right)_{\mathscr{L}^{2}[0,1]}=\int_{0}^{1} y_{i}(x) y_{0, i}(x) d x=1, \quad i \geqq 0 .
$$

Multiplying both parts of Eq. (I.11) by $y_{0, i}$ we get

$$
1=\mu_{i}\left(\mathbb{G}_{0} y_{i}, y_{0, i}\right)+\mu_{i}\left(\frac{\mathbb{G}_{1}}{\mathscr{L}^{1 / 2}} y_{i}, y_{0, i}\right), \quad i \geqq 0 .
$$

But since $y_{0, i}=\mu_{0, i} \mathbb{G}_{0} y_{0, i}$, therefore

$$
1=\frac{\mu_{i}}{\mu_{0, i}}+\mu_{i}\left(\frac{\mathbb{G}_{1}}{\mathscr{L}^{1 / 2}} y_{i}, y_{0, i}\right), \quad i \geqq 0 .
$$

Excluding $\mu_{i}$ from (I.12) we get

$$
\mu_{i}=\frac{\mu_{0, i}}{1+\mu_{0, i}\left(\frac{\mathbb{G}_{1}}{\mathscr{L}^{1 / 2}} y_{i}, y_{0, i}\right)}, \quad i \geqq 0 .
$$

Then from (I.11) and (I.13) we get the expression for $y_{i}$ :

$$
y_{i}=\frac{\mu_{0, i}}{1+\mu_{0, i}\left(\frac{\mathbb{G}_{1}}{\mathscr{L}^{1 / 2}} y_{i}, y_{0, i}\right)}\left(\mathbb{G}_{0}+\frac{\mathbb{G}_{1}}{\mathscr{L}^{1 / 2}}\right) y_{i}, \quad i \geqq 0,
$$

or

$$
y_{i}=\mu_{0, i}\left(\mathbb{G}_{0}+\frac{\mathbb{G}_{1}}{\mathscr{L}^{1 / 2}}\right) y_{i}-\mu_{0, i}\left(\frac{\mathbb{G}_{1}}{\mathscr{L}^{1 / 2}} y_{i}, y_{0, i}\right) y_{i}, \quad i \geqq 0
$$

and finally

$$
\left(E-\mu_{0, i} \mathbb{G}_{0}\right) y_{i}=\frac{\mu_{0, i}}{\mathscr{L}^{1 / 2}}\left(\mathbb{G}_{1} y_{i}-\left(\mathbb{G}_{1} y_{i}, y_{0, i}\right) y_{i}, \quad i \geqq 0\right.
$$

The operator $\left(E-\mu_{0, i} \mathbb{G}_{0}\right)$ is invertible in the subspace of functions which are orthogonal to $y_{0, i}$ and besides

$$
\left\|\left(E-\mu_{0, i} \mathbb{G}_{0}\right)^{-1}\right\| \leqq c_{i}, \quad i \geqq 0 .
$$

Therefore from (1.14) we obtain

$$
\begin{gathered}
y_{i}=y_{0, i}+\left(E-\mu_{0, i} \mathbb{G}_{0}\right)^{-1} \frac{\mu_{0, i}}{\mathscr{L}^{1 / 2}}\left(\mathbb{G}_{1} y_{i}-\left(\mathbb{G}_{1} y_{i}, y_{0, i}\right) y_{i}\right) \\
=T_{\mathscr{L}}^{(i)}(\omega) y_{i}, \quad i \geqq 0 .
\end{gathered}
$$


The operator $T_{\mathscr{L}}^{(i)}(\omega)$ on the set $A_{\mathscr{L}}(\varepsilon)$ transfers the ball $\left\|y_{i}-y_{0, i}\right\| \leqq 1$ to itself if

$$
\varrho=\frac{c_{i} \mu_{0, i}}{\mathscr{L}^{1 / 2}}(2 c(\varepsilon)+4 c(\varepsilon))=\frac{6 c(\varepsilon) c_{i} \mu_{0, i}}{\mathscr{L}^{1 / 2}} \leqq \frac{c_{1}(i, \varepsilon)}{\mathscr{L}^{1 / 2}}<1
$$

for all $i=0,1,2 \ldots(k-1)$ and $\mathscr{L}>c_{2}(k, \varepsilon)$. Moreover in this sphere this operator is a contraction with a contracting coefficient $\varrho$. Therefore it is possible to solve Eq. (I.15) by iterations taking as the first term $y_{0, i}, i=0,1,2 \ldots(k-1)$. Thus we get

$$
\begin{aligned}
y_{i}= & y_{0, i}+\frac{\mu_{0, i}}{\mathscr{L}^{1 / 2}}\left(E-\mu_{0, i} \mathbb{G}_{0}\right)^{-1}\left(\mathbb{G}_{1} y_{0, i}-\left(\mathbb{G}_{1} y_{0, i}, y_{0, i}\right) y_{0, i}\right)+R_{i, \mathscr{L}} ; \\
& \left\|R_{i, \mathscr{L}}\right\| \leqq \varrho^{2} \leqq \frac{c_{1}^{2}(i, \varepsilon)}{\mathscr{L}} .
\end{aligned}
$$

From (I.13) using (I.16) we obtain the expression for $\mu_{0, i}, i=0,1,2 \ldots(k-1)$;

$$
\begin{aligned}
\mu_{i} & =\frac{\mu_{0, i}}{1+\mu_{0, i}\left(\frac{\mathbb{G}_{1}}{\mathscr{L}^{1 / 2}} y_{i}, y_{0, i}\right)}=\mu_{0, i}\left(1+\frac{\mu_{0, i}}{\mathscr{L}^{1 / 2}}\left(\mathbb{G}_{1} y_{i}, y_{0, i}\right)\right)^{-1} \\
& =\mu_{0, i}-\frac{\mu_{0, i}^{2}}{\mathscr{L}^{1 / 2}}\left(\mathbb{G}_{1} y_{0, i}, y_{0, i}\right)+r_{i, \mathscr{L}}
\end{aligned}
$$

where

$$
\left|r_{i, \mathscr{L}}\right|<\frac{c_{3}(i, \varepsilon)}{\mathscr{L}}
$$

Letting $c_{i}^{(\mathscr{L})}(\omega)=-\mu_{0, i}^{2}\left(\mathbb{G}_{1} y_{0, i}, y_{0, i}\right)$ we get from (I.17)

$$
\mu_{i}=\mu_{0, i}+\frac{c_{i}^{(\mathscr{L})}(\omega)}{\mathscr{L}^{1 / 2}}+r_{i, \mathscr{L}} ; \quad\left|r_{i, \mathscr{L}}\right| \leqq \frac{c_{3}(i, \varepsilon)}{\mathscr{L}}
$$

Now we shall obtain evident formulae for the random variables $c_{i}^{(\mathscr{L})}(\omega)$, $i=0,1, \ldots(k-1)$.

As

$$
\left(\mathbb{G}_{1} y_{0, i}, y_{0, i}\right)=\int_{0}^{1} y_{0, i}(x) \int_{0}^{x} y_{0, i}(x) \xi_{\mathscr{L}}(z) d z d x+\int_{0}^{1} y_{0, i}(x) \xi_{\mathscr{L}}(x) \int_{x}^{1} y_{0, i}(z) d z d x
$$

Then using (I.10) we get

$$
\begin{aligned}
c_{i}^{(\mathscr{L})}(\omega)= & -\frac{2 \beta_{i}^{4}}{\alpha^{2}}\left(\int_{0}^{1} \sin \left(\beta_{i} x\right) \int_{0}^{x} \sin \left(\beta_{i} z\right) \xi_{\mathscr{L}}(z) d z d x\right. \\
& \left.+\int_{0}^{1} \sin \left(\beta_{i} x\right) \xi_{\mathscr{L}}(x) \int_{x}^{1} \sin \left(\beta_{i} z\right) d z d x\right) .
\end{aligned}
$$

Notice that under condition 2 a limit distribution of the random vector $\left\{c_{i}^{(\mathscr{L})}(\omega)\right\}$, $i=0,1, \ldots(k-1)$ is Gaussian and coincides with the distribution of the vector

$$
\begin{aligned}
& \left\{-\frac{2 \beta_{i}^{4}}{\alpha^{2}}\left(\int_{0}^{1} \sin \left(\beta_{i} x\right) \int_{0}^{x} \sin \left(\beta_{i} z\right) w(z) d z d x\right.\right. \\
& \left.\left.+\int_{0}^{1} \sin \left(\beta_{i} x\right) w(x) \int_{x}^{1} \sin \left(\beta_{i} z\right) d z d x\right)\right\}, \quad i=0,1,2 \ldots(k-1) .
\end{aligned}
$$


It is not difficult to obtain asymptotic formulas for the mean and the variance of the random variables $c_{i}^{(\mathscr{L})}(\omega), i=0,1 \ldots k-1$ and also to get the covariance matrix. We don't write out here these formulas, only notice that in general $b_{i j} \neq 0, i \neq j$.

Now letting $\zeta_{i}(\mathscr{L}, \omega)=\frac{\alpha}{\beta_{i}^{2}}\left(c_{i}^{(\mathscr{L})}(\omega)+r_{i, \mathscr{L}} \cdot \mathscr{L}^{1 / 2}\right),\left|r_{i \mathscr{L}}\right|<\frac{c_{3}(i, \varepsilon)}{\mathscr{L}}$, we get the statement of the theorem. Namely

$$
\mu_{i}=\mu_{0, i}+\frac{c_{i}^{(\mathscr{L})}(\omega)}{\mathscr{L}^{1 / 2}}+r_{i, \mathscr{L}}=\frac{\beta_{i}^{2}}{\alpha}\left[1+\frac{\zeta_{i}(\mathscr{L}, \omega)}{\mathscr{L}^{1 / 2}}\right], \quad i=0,1 \ldots(k-1),
$$

and consequently

$$
\begin{aligned}
\lambda_{i}(\mathscr{L}, \omega) & =\frac{\mu_{i}(\mathscr{L}, \omega)}{\mathscr{L}^{2}}=\frac{\beta_{i}^{2}}{\alpha \mathscr{L}^{2}}\left[1+\frac{\zeta_{i}(\mathscr{L}, \omega)}{\mathscr{L}^{1 / 2}}\right] \\
& =\frac{\pi^{2}(1+2 i)^{2}}{4 \alpha \mathscr{L}^{2}}\left[1+\frac{\zeta_{i}(\mathscr{L}, \omega)}{\mathscr{L}^{1 / 2}}\right], \quad i=0,1,2 \ldots(k-1)
\end{aligned}
$$

Now we shall give some examples where conditions 1 and 2 are fulfilled and the variance of the limit Wiener process is explicitly calculated.

Example $I$ is the most interesting since it generalize the well-known results [8] for the case of processes with continuous time.

Example I. Let $K$ be a compact metric space, $x_{t}(\omega)$ be a homogeneous Feller-Markov process on $K$ which is ergodic in the following sense: for any initial distribution $v$ on the $\sigma$-algebra $\mathscr{B}(K)$

$$
\int v(d x) P(t, x, \cdot) \Rightarrow \pi(\cdot), \quad t \rightarrow \infty .
$$

where $P(t, x, \Gamma)$ is the transition function of $x_{t}(\omega), \pi$ is the probability measure which is obviously the stationary distribution for this process. The convergence $\Rightarrow$ is weak, so the condition (I.16) is essentially weaker than the classical Doeblin condition [9].

Now let $f \in \mathscr{L}^{2}(\pi)$. Suppose that the equation

$$
A g=f
$$

has a unique solution belonging to $\mathscr{L}^{2}(\pi)$, where $A$ is the strong infinitesimal operator of the process $x_{t}(\omega)$. Note that from (I.16) it follows directly that

$$
\int f d \pi=(\pi, f)=0 \text {. }
$$

Proposition I. Let $x_{t}(\omega)$ be a stationary Markov process (i.e. an initial process with one-dimensional distribution $\pi$ ). Then the normalized functional

$$
\xi_{s}^{(\mathscr{L})}=\frac{1}{\mathscr{L}^{1 / 2}} \int f\left(x_{u}\right) d u, \quad s \in[0,1]
$$

converges to the Wiener process with the variance $\sigma^{2}=-2(A g, g)$ in the sense of Levy-Prohorov, that is in the sense of weak convergence of the distributions in $C[0,1]$. 
This proposition is similar to the Gordin-Lifšic theorem [8] and like the latter is based upon the principle of invariance for martingales [10]. Here we give only an outline of the arguments.

1. A process $\zeta_{t}=g\left(x_{t}\right)-\int_{0}^{t} A g\left(x_{s} d s\right.$ is a martingale.

2. Increments $\eta_{n}=\zeta_{n \Delta t}-\zeta_{(n-1) \Delta t}$ form a stationary and ergodic martingaledifference sequence.

3. Since $g\left(x_{t}\right)$ is restricted in probability the limit distributions

$$
\frac{\xi_{t \mathscr{L}}}{\mathscr{L}^{1 / 2}} \text { and } \frac{1}{\mathscr{L}^{1 / 2}} \int_{0}^{\mathscr{L}_{t}} A g\left(x_{s}\right) d s
$$

in $\mathrm{C}[0,1]$ coincide.

4. According to 2. all conditions of Billingsley's theorem were fulfilled, i.e. the successive sums of variables $\eta_{n}$ after a corresponding normalization converge (in the sense of Levy-Prohorov) to the Wiener process.

5. Let us find its parameters. We have

$$
\mathrm{D} \zeta_{n \Delta t}=n \mathrm{D} \eta_{1} \text {. }
$$

But

$$
\mathrm{D} \eta_{1}=\mathrm{M}_{\pi}\left(g\left(x_{\Delta t}\right)-g\left(x_{0}\right)-\int_{0}^{\Delta t} A g\left(x_{s}\right) d s\right)^{2}=-2 \Delta t(A g, g)+o(\Delta t)
$$

and $n \Delta t=t$ because

$$
\mathrm{D}\left(\frac{\zeta_{t}}{t^{1 / 2}}\right)=-2(A g, g)+o(1)
$$

and consequently

$$
\mathrm{D}\left(\frac{\zeta_{\mathscr{L} t}}{\mathscr{L}^{1 / 2}}\right) \sim-2(A g, g) t, \quad \mathscr{L} \rightarrow+\infty
$$

The proposition is proved.

Example Ia. Let $K$ be a compact Riemannien manifold and $x_{t}(\omega)$ be a diffusion process on $K$ with generator $\Delta$ (Laplace-Beltrami operator). Then $\pi(d x)=\delta(d x)$ is the normalized Riemannian measure. Let $f: K \rightarrow R^{1}$ be a continuous function on $K$ and $\hat{f}=\int f \delta(d x)$ be its mean value. Then

$$
\int_{0}^{t} f\left(x_{s}\right) d s=\hat{f} t+\int_{0}^{t}(f-\hat{f})\left(x_{s}\right) d s .
$$

But by the Fredholm alternative the equation

$$
\Delta g=f-\hat{f}
$$

has the unique smooth solution in $\mathscr{L}^{2}(\delta)$. The limit variance of the functional $\frac{1}{t^{1 / 2}} \int_{0}^{t}(f-\hat{f})\left(x_{s}\right) d s$ has the form

$$
\sigma^{2}=\int_{K}(\Delta g, g) \sigma d x=\int_{K}(\nabla g, \nabla g)^{2} \sigma(d x)
$$


where $\nabla g$ is the Riemannian gradient $g$. In other words $\sigma^{2}$ coincides with the quadratic Dirichlet form of $g$.

Example $I b$. Let $K=S^{1}$ and $x_{t}(\omega)$ be a Markov chain, with continuous time on $S^{1}=[0, \pi]$ (points 0 and $\pi$ are identified) with the constant density $\lambda$ of jumps and with the distribution $d F((y-x) \bmod 1)$ at the moment of the first jump from point

$x$. Then $A f(x)=\lambda\left(-f(x)+\int_{S^{1}} d F(y-x) f(y)\right)$. It is evident that under natural conditions of irreducibility (see below) the process $x_{t}(\omega)$ is ergodic with respect to the invariant measure on $S^{1}$ (Lebesgue measure). Let

$$
\int_{S^{1}} f d x=0
$$

Let us solve the equation $A g=-f$. If

$$
f=\sum_{-\infty}^{+\infty} a_{n} \exp (2 \pi i n x), \quad g=\sum_{-\infty}^{+\infty} c_{n} \exp (2 \pi i n x),
$$

then it is easy to establish that

$$
c_{n}=\frac{a_{n}}{\lambda\left(1-\gamma_{n}\right)}, \quad n \neq 0
$$

where

$$
\gamma_{n}=\int_{0}^{1} \exp (2 \pi i n x) d F(x)
$$

are the Fourier coefficients of the measure $d F$. The irreducibility mentioned above means that $\left|\gamma_{n}\right|<1$. It is clear that for the fixed measure $d F$ (even if $\lim _{n \rightarrow \infty} \gamma_{n}=1$ ) and for all "sufficiently smooth" functions $f$ we can suppress the influence of the small denominators $\left(1-\gamma_{n}\right)$ and obtain a smooth solution $g$.

Let us emphasize that for the process $x_{t}(\omega)$ in Example Ib (for some singular distribution $F$ ) Doeblin's condition was not fulfilled. Example Ib is a certain modification of [II].

Example2. Let $a(t, \omega)$ be a generalized renewal process, i.e. a process equal to $\frac{1}{\zeta_{i}}$ on the intervals the length of which form a sequence $\left\{\xi_{i}\right\}$ of independent random variables with the common distribution. $\left\{\zeta_{i}\right\}$ is also a sequence of independent random variables with common distribution with mean equal to 0 and a finite variance. $\left\{\zeta_{i}\right\}$ and $\left\{\xi_{i}\right\}$ are independent. Then using the Donsker-Prohorov theorem for $\int_{0}^{t \mathscr{L}} \frac{d s}{a(s, \omega)}$ it is easy to show that the distribution of the process $\mathscr{L}^{-1 / 2} \int_{0}^{t \mathscr{L}} \frac{d s}{a(s, \omega)}$ weakly converges to the distribution of a Wiener process with variance $\sigma^{2}=\mathrm{D}\left(\zeta_{i} \zeta_{i}\right)$. 


\section{The "Pieces" Model}

In this section we consider the simplest potential model, the so-called "pieces" model as the first approach to a model with generalized $\delta$-potential whose hamiltonian has the form

$$
H(\omega)=-\frac{d^{2}}{d t^{2}}+\sum_{i=-\infty}^{+\infty} \xi_{i}(\omega) \delta\left(t-\tau_{i}\right)
$$

where $\tau_{i}$ is a Poisson flow of points and $\xi_{i}(\omega)$ are independent random variables. Roughly speaking in the "pieces" model $\xi_{i} \equiv \infty$. The more exact definition is the following: let $\tau_{i}, i=1,2, \ldots v_{\mathscr{L}}$ be the points of Poisson flow with parameter $\lambda$ concentrated on $[0, \mathscr{L}], v_{\mathscr{L}}$ be the number of points of the flow in $[0, \mathscr{L}]$. Let us consider the spectral problem

$$
-\frac{d^{2}}{d t^{2}} y=\lambda y, \quad y(0)=y(\mathscr{L})=0,
$$

with the constraints $y\left(\tau_{i}\right)=0, \tau_{i} \in[0, \mathscr{L}]$. This problem decomposes in fact into a set of spectral problems without potential on each piece $\Delta_{1}, \Delta_{2}, \ldots \Delta_{v_{\mathscr{L}}+1}$, bounded by the points of the flow. If for example $v_{\mathscr{L}}=n$, then our problem is a set of $n+1$ boundary problems with zero boundary conditions on every interval of the partition. The eigenvalues of the restriction of the operator $H(\omega)$ to the $i^{\text {th }}$ interval of the partition are equal to $\lambda_{i, m}=\frac{\pi^{2} m^{2}}{\Delta_{i}^{2}}, m \in N$, where $\Delta_{i}$ is a length of $i^{\text {th }}$ interval, and consequently, the spectrum of the operator $H(\omega)$ is equal to $\bigcup_{i=1}^{n+1} \bigcup_{m \in N}\left\{\frac{\pi^{2} m^{2}}{\Delta_{i}^{2}}\right\}$. We are interested in the structure of the spectrum at the left end. It follows directly from the above given formulae that the study of the distances between the first eigenvalues may be reduced to the study of the sequence $\Delta_{i}, i=1,2 \ldots(n+1)$. Actually the minimal eigenvalue of the operator $H(\omega)$ is equal to $\lambda_{1}=\frac{\pi^{2}}{\left(\max _{\Delta_{i} \in[0, \mathscr{L}]} \Delta_{i}\right)^{2}}$ and if $\Delta_{n+1} \geqq \Delta_{(n)} \geqq \ldots \geqq \Delta_{(1)}$ is the ordered set of $\left\{\Delta_{i}\right\}$ in increasing order then

$$
\lambda_{2}=\frac{\pi^{2}}{\Delta_{(n)}^{2}} ; \lambda_{3}=\frac{\pi^{2}}{\Delta_{(n-1)}^{2}} \ldots \lambda_{c}=\frac{\pi^{2}}{\Delta_{(n-k+2)}^{2}}, \quad k<n .^{2}
$$

Now let us consider the interval $[0, \mathscr{L}]$ and the sequence of intervals $\Delta_{1}$, $\Delta_{2}, \ldots \Delta_{v \mathscr{L}+1}$. It is known that the random variables $\Delta_{1}, \Delta_{2}, \ldots \Delta_{v \mathscr{L}+1}$ have the same exponential distribution. For the sake of simplicity we assume that the parameter of the exponential distribution $\lambda=1$. The random variable $v_{\mathscr{L}}$ has a Poisson distribution with mean $\mathscr{L}[15]\left(\right.$ i.e. $\left.P\left\{v_{\mathscr{L}}=n\right\}=\exp (-\mathscr{L}) \frac{\mathscr{L}^{n}}{n !}\right)$; besides, from the

2 We use the fact that when $\mathscr{L} \rightarrow+\infty$ and $k \leqq k_{0}$ the senior values $\Delta_{(n+1)}, \Delta_{(n)}, \ldots \Delta_{(n-k+2)}$ are equivalent in probability so that e.g. the second eigenvalue on the interval $\Delta_{(n+1)}$ is greater with probability approaching 1 than the first values on $\Delta_{(n)}, \Delta_{(n-1)}, \ldots \Delta_{(n-k+2)}$ (see below) 
central limit theorem it follows that $v_{\mathscr{L}}=\mathscr{L}+\mathscr{L}^{1 / 2} \xi$, when $\mathscr{L} \rightarrow \infty$, where $\xi$ is a random variable having asymptotically normal distribution with parameters $(0, \mathrm{I})$.

Let us list several properties of the exponential distribution which will be used in the proof of the main theorem concerning the distribution of the first eigenvalues of the random operator $H(\omega)$ (for proofs see [15]).

Proposition I. Under the condition $v_{\mathscr{L}}=n$ the interval $[0, \mathscr{L}]$ is decomposed into $n+1$ intervals by random points $\tau_{i}, i=1,2 \ldots n$ and the distribution of the vector of lengths $\left(\Delta_{1}, \Delta_{2} \ldots \Delta_{n+1}\right)$, coincides with the distribution of the random vector

$$
\begin{gathered}
\left.\mathscr{L} \cdot \frac{\eta_{1}}{\eta_{1}+\ldots+\eta_{n+1}}, \quad \mathscr{L} \cdot \frac{\eta_{2}}{\eta_{1}+\ldots+\eta_{n+1}}, \ldots \mathscr{L} \cdot \frac{\eta_{n+1}}{\eta_{1}+\eta_{2}+\ldots+\eta_{n+1}}\right), \text { where } \eta_{i}, \\
i=1,2, \ldots(n+1)
\end{gathered}
$$

are independent random variables having common exponential distribution with parameter $\lambda=1$.

We can write

$$
\Delta_{i}=\mathscr{L} \cdot \frac{\eta_{i}}{\eta_{1}+\eta_{2}+\ldots+\eta_{n+1}}, \quad i=1,2, \ldots(n+1) .
$$

Thus from Proposition 1 it follows that the study of lengths of the random intervals is reduced to the study of the sequence of independent identically distributed random variables.

Proposition 2. Let $\eta_{1}, \eta_{2} \ldots \eta_{n}$ be independent random variables having the same exponential distribution with parameter 1 . Let $\eta_{(n)} \geqq \eta_{(n-1)} \geqq \ldots \geqq \eta_{(1)}$ be the ordered sample of $\eta_{1}, \eta_{2} \ldots \eta_{n}$. Then the joint distribution density of the random variables

$$
\eta_{(n)}-\eta_{(n-1)}, \eta_{(n-1)}-\eta_{(n-2)} \ldots \eta_{(n-k+1)}-\ln n, \quad k<n,
$$

when $n \rightarrow \infty$ is asymptotically equal to

$$
\exp \left(-y_{1}-2 y_{2}-\ldots-k y_{k}-\exp \left(-y_{k}\right)\right)
$$

where $y_{1}, y_{2} \ldots y_{k-1}>0, y_{k} \in R^{1}$.

Proposition 3. Let $v_{\mathscr{L}}=n$. If

$$
\xi^{(n+1)}=-\frac{n+1}{\mathscr{L}}\left(\Delta_{(n+1)}-\frac{\mathscr{L} \ln (n+1)}{n+1}\right),
$$

or

$$
\xi^{(n+1)}=-\frac{n+1}{\mathscr{L}} \Delta_{(n+1)}+\ln (n+1),
$$

then

$$
\lim _{n \rightarrow \infty} P\left\{\xi^{(n+1)} \leqq x\right\}=\exp (-\exp (-x))
$$

i.e. $\xi^{(n+1)}$ has a double exponential distribution in the limit $n \rightarrow \infty$. 
Proposition 4. The arguments used in Proposition 3 are applicable to the sequence of lengths

$$
\begin{aligned}
\Delta_{(n+1)} & =\mathscr{L} \frac{\eta_{(n+1)}}{\eta_{1}+\eta_{2}+\ldots+\eta_{n+1}}, \\
\Delta_{(n)} & =\mathscr{L} \frac{\eta_{(n)}}{\eta_{1}+\eta_{2}+\ldots+\eta_{n+1}} \ldots \Delta_{(n-k+2)}=\mathscr{L} \frac{\eta_{(n-k+2)}}{\eta_{1}+\eta_{2}+\ldots+\eta_{n+1}},
\end{aligned}
$$

and as a result we obtain that there exists a limit distribution for

$$
\frac{n+1}{\mathscr{L}} \Delta_{(n-i)}-\ln (n+1), \quad n \rightarrow \infty .
$$

We assume now that the number of points $v_{\mathscr{L}}$ in the interval $[0, \mathscr{L}]$ is not fixed. As was mentioned above, the random variable $v_{\mathscr{L}}$ has a Poisson distribution with parameter $\mathscr{L}$ and $v_{\mathscr{L}}=\mathscr{L}+\mathscr{L}^{1 / 2} \xi$, where the random variable $\xi$ is asymptotically normal. So we obtain from Proposition 4 that if

$$
\Delta_{\left(v_{\mathscr{L}}+1\right)} \geqq \Delta_{\left(v_{\mathscr{L}}\right)} \geqq \ldots \geqq \Delta_{\left(v_{\mathscr{L}}-k+2\right)},
$$

then for the random variables $\xi^{(i)}(\mathscr{L}, \omega)=-\Delta_{(v \mathscr{L}-i+2)}+\ln \mathscr{L}, i=1,2 \ldots k$ the limit distribution exists when $\mathscr{L} \rightarrow \infty$.

Let us return to the study of the first $k$ eigenvalues $\lambda_{1}, \lambda_{2} \ldots \lambda_{k}$ of the operator $H(\omega)$. As was stated earlier

$$
\lambda_{i}(\mathscr{L}, \omega)=\frac{\pi^{2}}{\Delta_{(v \mathscr{L}+2-i)}^{2}},
$$

but $\Delta_{(v \mathscr{L}+2-i)}=\ln \mathscr{L}-\xi^{(i)}(\mathscr{L}, \omega)$, therefore

$$
\lambda_{i}(\mathscr{L}, \omega)=\frac{\pi^{2}}{\ln ^{2} \mathscr{L}}+\frac{2 \pi^{2} \xi^{(i)}(\mathscr{L}, \omega)}{\ln ^{3} \mathscr{L}}+O\left(\frac{1}{\ln ^{4} \mathscr{L}}\right),
$$

when $\mathscr{L} \rightarrow \infty$. Let us put

$$
\begin{gathered}
\xi^{(i)}(\mathscr{L}, \omega)=\frac{-\ln ^{3} \mathscr{L}}{2 \pi^{2}}\left(\lambda_{i}-\frac{\pi^{2}}{\ln ^{2} \mathscr{L}}\right) ;-\zeta^{(i)}(\mathscr{L}, \omega) \sim^{(p)} \xi^{(i)}(\mathscr{L}, \omega) \\
\lambda_{i}(\mathscr{L}, \omega)=\frac{\pi^{2}}{\ln ^{2} \mathscr{L}}\left(1-\frac{2 \zeta^{(i)}(\mathscr{L}, \omega)}{\ln \mathscr{L}}\right), \quad i=1,2 \ldots k
\end{gathered}
$$

Now using Proposition 2 it is easy to show that the random variables

$$
\zeta^{(1)}(\mathscr{L}, \omega)-\zeta^{(2)}(\mathscr{L}, \omega), \ldots \zeta^{(k-1)}(\mathscr{L}, \omega)-\zeta^{(k)}(\mathscr{L}, \omega), \zeta^{(k)}(\mathscr{L}, \omega)
$$

are asymptotically independent when $\mathscr{L} \rightarrow \infty$ and have the following joint distribution density

$$
\begin{gathered}
p\left(y_{1}, y_{2} \ldots y_{x}\right)=\exp \left(-y_{1}-2 y_{2}-\ldots-x y_{k}-\exp \left(-y_{k}\right)\right) \\
y_{1}, y_{2} \ldots y_{k-1}>0, y_{k} \in R^{1},
\end{gathered}
$$

or, which is the same, random variables

$$
\zeta^{(x)}(\mathscr{L}, \omega),-\left(\zeta^{(x)}(\mathscr{L}, \omega)-\zeta^{(x-1)}(\mathscr{L}, \omega)\right), \ldots-\left(\zeta^{(2)}(\mathscr{L}, \omega)-\zeta^{(1)}(\mathscr{L}, \omega)\right)
$$


have the following limit density

$$
\begin{aligned}
p_{x}(x) & =\frac{1}{(x-1) !} \exp (-k x-\exp (-x)), p_{k-1}(x) \\
& =(k-1) \exp (-(k-1) x) \chi_{\{x \geqq 0\}} \ldots p_{1}(x)=\exp (-x) \chi_{\{x \geqq 0\}} .
\end{aligned}
$$

Thus we have proved the following theorem

Theorem 2. In the "pieces" model for the fixed $k$ and $\mathscr{L} \rightarrow+\infty$ the following statement is true:

$$
\lambda_{i}(\mathscr{L}, \omega)=\frac{\pi^{2}}{\ln ^{2} \mathscr{L}}\left(1-\frac{2 \zeta^{(i)}(\mathscr{L}, \omega)}{\ln \mathscr{L}}\right), \quad i=1,2 \ldots k,
$$

where the random variables $\zeta^{(i)}(\mathscr{L}, \omega)$ have such limit distributions that random variables

$$
\zeta^{(k)}(\mathscr{L}),-\left(\zeta^{(k)}(\mathscr{L})-\zeta^{(k-1)}(\mathscr{L})\right) \ldots-\left(\zeta^{(2)}(\mathscr{L})-\zeta^{(1)}(\mathscr{L})\right)
$$

are asymptotically independent and have limit densities

$$
\begin{aligned}
p_{k}(x) & =\frac{1}{(x-1) !} \exp (-k x-\exp (-x)), p_{k-1}(x) \\
& =(k-1) \exp (-(x-1) k) \chi_{\{x \geqq 0\}} \ldots p_{1}(x)=\exp (-x) \chi_{\{x \geqq 0\}} .
\end{aligned}
$$

While proving Theorem 1 we have also proved that the eigenfunctions $y_{1}, y_{2} \ldots y_{k}$ in the "pieces" model are localized on an interval of the length $O(\ln \mathscr{L})$.

\section{3. $\delta$-Potential}

Let us consider the following boundary problem on the interval $[0, \mathscr{L}]$ :

$$
\begin{aligned}
H y=-\frac{d^{2}}{d t^{2}} y+\mathscr{V}(t, \omega) y & \equiv \frac{d^{2} y}{d t^{2}}+\sum_{\tau_{i} \in[0, \mathscr{L}]} \xi_{i} \delta\left(t-\tau_{i}\right) y=\lambda y, \\
y(0) & =y(\mathscr{L})=0,
\end{aligned}
$$

where the $\tau_{i}$ are the points of a Poisson flow with parameter $1,\left\{\tau_{i}\right\}$ do not depend on $\xi_{i}$ and $\xi_{i}$ are independent random variables with the common distribution

$$
P\left\{\xi_{i}>x\right\}=\left\{\begin{array}{l}
1, \quad x \leqq 0 \\
\exp (-\mu x), \quad x>0, \quad \mu \geqq 0 .
\end{array}\right.
$$

Let us introduce as usual [4] the amplitude and phase by the formulae

$$
\left\{\begin{array}{l}
y=r \sin (\theta(t)) \\
y^{\prime}=r \cos (\theta(t))
\end{array}\right.
$$

It is known that a problem (3.1) is reduced to the following boundary problem for the phase

$$
\frac{d \theta(t)}{d t}=\cos ^{2}(\theta(t))+(\lambda-\mathscr{V}(t, \omega)) \sin ^{2}(\theta(t)) .
$$


The equations for the first eigenvalue have the form

$$
\theta_{\lambda}(0)=0, \theta_{\lambda}(\mathscr{L})=\pi \text {. }
$$

For the second $\lambda_{2}$ it has the form

$$
\theta_{\lambda}(0)=0, \theta_{\lambda}(\mathscr{L})=2 \pi
$$

and so on.

Thus we get the following picture. The random variable $\theta(t)$ changes inside the interval $[0, \pi]$ according to Eq. (3.4). At the initial moment $t=0$ it is at point 0 . Then its motion consists of the determined motion with the speed $\cos ^{2}(\theta(t))$ $+\lambda \sin ^{2}(\theta(t))$ towards $\pi$ and random jumps in the opposite direction determined by the random potential $\mathscr{V}(t, \omega)$. The minimal $\lambda$ for which the phase $\theta_{\lambda}(\mathscr{L})$ equals $\pi$ coincides with the first eigenvalue $\lambda_{1}$ of the boundary problem (3.1). The equation $\theta_{\lambda}(\mathscr{L})=2 \pi$ defines the second eigenvalue $\lambda_{2}$ of the problem (3.1) and so on. It is convenient to identify intervals $[\pi k, \pi(k+1)]$ with the interval $[0, \pi]$ and to imagine that the process instantly jumps from the point $\pi$ to the point 0 . Notice that the process $\theta(t)$ can return to the point 0 only after passing through the point $\pi$ since $\frac{d \theta(0)}{d t}=1$. It is technically simpler to deal with the whole axis $(-\infty,+\infty)$ rather than with the interval $[0, \pi]$. We make the following change of variables

$$
x(t)=-\operatorname{ctg}(\theta(t)), \quad t \geqq 0 .
$$

Then for $x(t)$ we obtain the Riccati equation

$$
\frac{d x}{d t}=x^{2}+\lambda-\mathscr{V}(t, \omega)
$$

from which the Markov property of the process $x(t)$ on the whole real axis follows. We shall calculate its infinitesimal operator $A$. Let our process at the initial moment be equal to $x$. For a short interval of time $\Delta t$ it has no jumps with probability $1-\Delta t+o(\Delta t)$ and under this condition it passes to the point

$$
x(\Delta t)=x+\left(x^{2}+\lambda\right) \Delta t+o(\Delta t) .
$$

With probability $\Delta t+o(\Delta t)$ a jump occurs and then the process $x(t)$ is equal to

$$
x(\Delta t)=x-\xi+o(\Delta t),
$$

where $\xi$ has distribution (3.2). Let us take now an arbitrary smooth function $f(u)$ and calculate the mean value

$$
\frac{M_{x} f(x(\Delta t))-f(x)}{\Delta t} .
$$

From (3.8) and (3.9) it follows immediately that

$$
\begin{aligned}
A f(x)= & \lim _{\Delta t \rightarrow 0} \frac{\mathrm{M}_{x} f(x(\Delta t))-f(x)}{\Delta t}=-f(x)+f^{\prime}(x)\left(x^{2}+\lambda\right) \\
& +\mu \int_{0}^{\infty} f(x-y) \exp (-\mu y) d y .
\end{aligned}
$$


Of particular importance here is $\tau_{\lambda}(x)$, which is equal to the first passage time to $+\infty$ under the condition $x(0)=x$;

$$
\tau_{\lambda}(x)=\min _{t}\{t: x(t)=+\infty \mid x(0)=x\} .
$$

From what was said above it is clear that the condition

$$
\tau_{\lambda}(-\infty)>\mathscr{L}
$$

is equivalent to the condition

$$
\lambda_{1}>\lambda
$$

(the condition $\tau_{\lambda}^{(1)}+\tau_{\lambda}^{(2)}>\mathscr{L}^{3}$ is equivalent to the condition $\lambda_{2}>\lambda$ etc.).

So, if we know the asymptotic distribution of $\tau_{\lambda}(-\infty)$ when $\lambda \rightarrow 0$ we obtain the asymptotic distribution of the first eigenvalue of (3.1). In order to get this distribution we use the method of moments. Let

$$
u(x, \alpha)=M_{x}\left(\exp \left(-\alpha \tau_{\lambda}(x)\right)\right)
$$

From the general theory of Markov processes it follows that the function (3.14) is a solution of the problem

$$
A u-\alpha u=0, u(+\infty)=1,
$$

where $A$ is the infinitesimal operator of our process $x(t)$ given by (3.10).

Thus we come to a boundary problem which looks as follows:

$$
\begin{gathered}
\left(x^{2}+\lambda\right) \frac{d u(x)}{d x}+\mu \int_{0}^{+\infty} u(x-y) \exp (-\mu y) d y-(1+\alpha) u(x)=0, \\
u(+\infty)=1 .
\end{gathered}
$$

Let us introduce a new function $v(x)$ putting

$$
v(x)=\mu \int_{0}^{+\infty} u(x-y) \exp (-\mu y) d y=\mu \exp (-\mu x) \int_{-\infty}^{x} u(t) \exp (\mu t) d t
$$

For function $u(x)$ and $v(x)$ we get the following boundary problem

$$
\left\{\begin{array}{l}
\left(x^{2}+\lambda\right) \frac{d u}{d x}+v-(1+\alpha) u=0 \\
\frac{d v}{d x}=\mu(u-v) \\
u(+\infty)=v(+\infty)=1 .
\end{array}\right.
$$

Excluding $v(x)$ from (3.18) we get

$$
\begin{gathered}
\left(x^{2}+\lambda\right) \frac{d^{2} u}{d x^{2}}+\left(\mu x^{2}+2 x+\mu \lambda-1-\alpha\right) \frac{d u}{d x}-\mu \alpha u=0, \\
u(+\infty)=1, u^{\prime}(+\infty)=0 .
\end{gathered}
$$

$3 \tau_{\lambda}^{(i)}$ is the time of $i^{\text {th }}$ phase revolution, i.e. transition of process $x(t)$ from $-\infty$ to $+\infty$ 
Let us introduce the functions

$$
u_{k}(x)=\frac{\partial^{k} u(x, 0)}{\partial \alpha^{k}}
$$

It is easy to see that

$$
\begin{aligned}
& u_{0}(x) \equiv 1, \\
& u_{k}(x)=(-1)^{k} \mathrm{M}\left\{\tau_{\lambda}^{(k)}(x)\right\}, \quad k \geqq 1 .
\end{aligned}
$$

Differentiating (3.19) with respect to $\alpha$ and supposing $\alpha=0$ we come to the system of equations

$$
\begin{gathered}
\left(x^{2}+\lambda\right) \frac{\partial^{2} u_{k}}{\partial x^{2}}+\left(\mu x^{2}+2 x+\mu \lambda-1\right) \frac{\partial u_{k}}{\partial x}-x\left(\frac{\partial u_{k-1}}{\partial x}+\mu u_{k-1}\right)=0, \\
u_{k}(+\infty)=0, u_{k}^{\prime}(+\infty)=0, \quad k \geqq 1 .
\end{gathered}
$$

Note that any $u_{k}(x)$ is expressed through $u_{k-1}(x)$ in view of (3.22). This allows us to solve Eq. (3.22) recurrently for $k=1,2 \ldots$ (As for the boundary conditions in (3.22) they follow from the estimates

$$
\begin{gathered}
\mathrm{M}\left\{\tau_{\lambda}^{k}(x)\right\} \leqq c_{x}, \\
\lim _{x \rightarrow+\infty} \mathrm{M}\left\{\tau_{\lambda}^{k}(x)\right\}=0, \quad k=0,1,2 \ldots,
\end{gathered}
$$

where $c_{k}$ does not depend on $x$ for any $k$.)

The first estimate in (3.23) follows from the fact that $\tau_{\lambda}(x)$ is majorized by a small similar value in the "piece" model (see Sect. 2).

Integrating (3.23) we obtain

$$
u_{k}(x)=-k \int_{x}^{\infty} \frac{\exp (-\mu z+\varphi(z))}{z^{2}+\lambda} d z \int_{-\infty}^{z}\left[u_{k-1}^{\prime}(t)+\mu u_{k-1}(t)\right] \exp (\mu t-\varphi(t)) d t,
$$

where

$$
\varphi(t)=\frac{1}{\lambda^{1 / 2}} \operatorname{aretg}\left(\frac{t}{\lambda^{1 / 2}}\right), \quad k=1,2 \ldots
$$

Integrating by parts in (3.24) we get the following expression for $u_{k}(x)$ :

$$
u_{k}(x)=-k \int_{x}^{\infty} \frac{u_{k-1}(z)}{z^{2}+\lambda} d z-k \int_{x}^{\infty} \frac{\exp (-\mu z+\varphi(z))}{z^{2}+\lambda} d z \int_{-\infty}^{z} \frac{\exp (\mu t-\varphi(t)) u_{k-1}(t)}{t^{2}+\lambda} d t .
$$

Theorem 3. For any $k=1,2 \ldots$ and any $x:-\infty \leqq x \leqq 0$ the asymptotic formula

$$
u_{k}(x) \sim(-1)^{k} k ! \exp \left(\pi k / \lambda^{1 / 2}\right)
$$

is valid when $\lambda \rightarrow+0$ (uniformly in $x$ ). 
Proof. We shall prove (3.26) by induction. Expression (3.26) is obviously true for $k=0$. Let now (3.26) be true for a $k-1$; we shall prove that it is equally true for $k$. Let us put

$$
g_{k-1}(z)=\int_{-\infty}^{z} \frac{\exp (\mu t-\varphi(t))}{t^{2}+\lambda} u_{k-1}(t) d t
$$

Now we shall estimate the second additional term in (3.25) when $x=-\infty$

$$
\begin{aligned}
& \int_{x}^{+\infty} \frac{\exp (-\mu z+\varphi(z))}{z^{2}+\lambda} g_{k-1}(z) d z=\int_{x}^{0} \frac{\exp (-\mu z+\varphi(z))}{z^{2}+\lambda} g_{k-1}(z) d z \\
& \quad+\int_{0}^{+\infty} \frac{\exp (-\mu z+\varphi(z))}{z^{2}+\lambda} g_{k-1}(z) d z .
\end{aligned}
$$

From the induction hypothesis

$$
\left|u_{k-1}(x)\right| \leqq\left|u_{k-1}(-\infty)\right| \leqq c \exp \left(\pi(k-1) / \lambda^{1 / 2}\right)
$$

for some $c$ and sufficiently small $\lambda$. Consequently from (3.27) and (3.29) we get the following estimate for $z \leqq 0$ :

$$
\begin{aligned}
\left|g_{k-1}(z)\right| & \leqq \int_{-\infty}^{z} \frac{\exp (\mu t-\varphi(t))}{t^{2}+\lambda}\left|u_{k-1}(t)\right| d t \\
& \leqq C \exp \left(\pi(k-1) / \lambda^{1 / 2}+\mu z\right)\left(\exp \left(\pi / 2 \lambda^{1 / 2}\right)-\exp (-\varphi(z))\right) \\
& \leqq c \exp \left(\mu z+\pi(k-1 / 2) / \lambda^{1 / 2}\right) .
\end{aligned}
$$

From (3.30) it follows that for the first term in the right-hand part of (3.28) the following inequality is true

$$
\begin{aligned}
& \left|\int_{x}^{0} \frac{\exp (-\mu z+\varphi(z))}{z^{2}+\lambda} g_{k-1}(z) d z\right| \leqq \int_{-\infty}^{0} \frac{\exp (-\mu z+\varphi(z))}{z^{2}+\lambda}\left|g_{k-1}(z)\right| d z \\
& \quad \leqq c \exp \left(\pi(k-1 / 2) / \lambda^{1 / 2}\right)
\end{aligned}
$$

In order to estimate the second term in (3.28) let us put

$$
g_{k-1}(z)=g_{k-1}(0)+\int_{0}^{z} \frac{\exp (\mu t-\varphi(t))}{t^{2}+\lambda} u_{k-1}(t) d t=g_{k-1}(0)+\Delta g_{k-1}
$$

when $z \geqq 0$. From (3.30) it follows that

$$
\left|\Delta g_{k-1}\right| \leqq c \exp \left(\mu z+\pi(k-1) / \lambda^{1 / 2}\right)
$$

and thus

$$
\left|\int_{0}^{\infty} \frac{\exp (-\mu z+\varphi(z))}{z^{2}+\lambda} \Delta g_{k-1} d z\right| \leqq c \exp \left(\pi(k-1 / 2) / \lambda^{1 / 2}\right)
$$

Then

$$
\begin{aligned}
& \int_{0}^{\infty} \frac{\exp (-\mu z+\varphi(z))}{z^{2}+\lambda} g_{k-1}(z) d z=g_{k-1}(0) \int_{0}^{\infty} \frac{\exp (-\mu z+\varphi(z))}{z^{2}+\lambda} d z \\
& \quad+\int_{0}^{\infty} \frac{\exp (-\mu z+\varphi(z))}{z^{2}+\lambda} \Delta g_{k-1} d z .
\end{aligned}
$$


But

$$
\begin{aligned}
& \int_{0}^{\infty} \frac{\exp (-\mu z+\varphi(z)) d z}{z^{2}+\lambda}=-1+\mu \int_{0}^{\infty} \exp (-\mu z+\varphi(z)) d z \\
& \quad=\int_{0}^{\infty} \exp \left(-z+\varphi\left(\frac{z}{\mu}\right)\right) d z-1 \\
& \quad=\exp \left(\pi / 2 \lambda^{1 / 2}\right) \int_{0}^{\infty} \exp \left(-z+\varphi\left(\frac{z}{\mu}\right)-\frac{\pi}{2 \lambda^{1 / 2}}\right) d z-1 \\
& \quad=\exp \left(\pi / 2 \lambda^{1 / 2}\right)[1+o(1)], \quad \lambda \rightarrow+0 .
\end{aligned}
$$

The last equality is true since it is obvious that

$$
\left|\exp \left(\varphi\left(\frac{z}{\mu}\right)-\frac{\pi}{2 \lambda^{1 / 2}}\right)\right| \leqq 1, \exp \left(\varphi\left(\frac{z}{\mu}\right)-\frac{\pi}{2 \lambda^{1 / 2}}\right) \rightarrow 1, \text { when } \lambda \rightarrow+0 .
$$

In its turn

$$
\begin{aligned}
g_{k-1}(0)= & \int_{-\infty}^{0} \frac{\exp (\mu t-\varphi(t))}{t^{2}+\lambda} u_{k-1}(t) d t \\
= & \int_{0}^{\infty} \frac{\exp (-\mu t+\varphi(t))}{t^{2}+\lambda} u_{k-1}(t) d t \\
= & (-1)^{k-1}(k-1) ! \exp \left(\pi(k-1) / \lambda^{1 / 2}\right) \int_{0}^{\infty} \frac{\exp (-\mu t+\varphi(t))}{t^{2}+\lambda} d t \\
& +\int_{0}^{\infty} \frac{\exp (-\mu t+\varphi(t))\left[u_{k-1}(-t)-(-1)^{k-1}(k-1) ! \exp \left(\pi(k-1) / \lambda^{1 / 2}\right)\right]}{t^{2}+\lambda} d t
\end{aligned}
$$

Assuming the induction

$$
u_{k-1}(-t)-(-1)^{k-1}(k-1) ! \exp \left(\pi(k-1) / \lambda^{1 / 2}\right)=o\left(\exp \left(\pi(k-1) / \lambda^{1 / 2}\right)\right), \quad \lambda \rightarrow+0
$$

Notice finally that from (3.29)

$$
\begin{aligned}
\left|\int_{x}^{\infty} \frac{u_{k-1}(z)}{z^{2}+\lambda} d z\right| & \leqq \int_{-\infty}^{+\infty} \frac{\left|u_{k-1}(z)\right|}{z^{2}+\lambda} d z \leqq c \cdot \pi \lambda^{-1 / 2} \exp \left(\pi(k-1) / \lambda^{1 / 2}\right) \\
& =o\left(\exp \left(\pi k / \lambda^{1 / 2}\right)\right), \quad \lambda \rightarrow+0 .
\end{aligned}
$$

And at last (3.27) follows from (3.25) and (3.31)-(3.38).

Thus Theorem 3 is proved completely.

Corollary I. In the limit $\lambda \rightarrow+0$ the random variable $\tilde{\tau}_{\lambda}=\frac{\tau_{\lambda}(-\infty)}{\exp \left(\pi / \lambda^{1 / 2}\right)}$ has an exponential distribution with parameter 1.

Actually, from (3.21) and (3.26) it follows that when $\lambda \rightarrow+0$

$$
\mathrm{M}\left\{\tilde{\tau}_{\lambda}^{k}\right\}=\mathrm{M}\left\{\left(\frac{\tau_{\lambda}(-\infty)}{\exp \left(\pi / \lambda^{1 / 2}\right)}\right)^{k}\right\} \sim k !
$$


But since the distribution is uniquely determined by all its moments

$$
P\left\{\tilde{\tau}_{\lambda}>x\right\} \underset{\lambda \rightarrow+0}{\longrightarrow}\left\{\begin{array}{l}
1, \quad x<0 \\
\exp (-x), \quad x \geqq 0 .
\end{array}\right.
$$

From Corollary I it is easy to get

Corollary 2. If $N(\lambda)=\sum_{\lambda_{i}(\mathscr{\mathscr { C }}) \leqq \lambda} 1$ then there exists a nonrandom continuous limit

$$
N(\lambda)=\lim _{\mathscr{L} \rightarrow+\infty} \frac{1}{\mathscr{L}} N_{\mathscr{L}}(\lambda),
$$

and

$$
N(\lambda) \sim \exp \left(-\pi / \lambda^{1 / 2}\right), \text { when } \lambda \rightarrow+0\left(\text { as } N(\lambda)=\left(M \tau_{\lambda}\right)^{-1}\right) .
$$

Notice that this result was obtained earlier in [3].

Let us connect parameters $\mathscr{L}$ and $\lambda$ by the expression

$$
\mathscr{L} \cdot \exp \left(-\pi / \lambda^{1 / 2}\right)=z,
$$

where $z$ is a fixed positive number. From (3.40) it follows that

$$
\lambda=\frac{\pi^{2}}{\ln ^{2} \mathscr{L}}\left(1-\frac{\ln z}{\ln \mathscr{L}}\right)^{-2}=\frac{\pi^{2}}{\ln ^{2} \mathscr{L}}+\frac{2 \pi^{2} \ln z}{\ln ^{3} \mathscr{L}}+O\left(\frac{1}{\ln ^{4} \mathscr{L}}\right) .
$$

Let us introduce the normalized eigenvalues of problem (3.1) in the following way:

$$
\tilde{\lambda}_{k}=\frac{\ln ^{3} \mathscr{L}}{2 \pi^{2}}\left(\lambda_{k}-\frac{\pi^{2}}{\ln ^{2} \mathscr{L}}\right) .
$$

Theorem 4. For any natural $k$ there exists a limit distribution of $\tilde{\lambda}_{k}$ (when $\mathscr{L} \rightarrow+\infty$ ) with density

$$
p_{k}(x)=\frac{1}{(k-1) !} \exp (k x-\exp (x)), x \in R^{1} .
$$

Proof. Taking into account (3.12), (3.13), (3.39), (3.41) and (3.42) we can write the following sequence of equalities

$$
\begin{aligned}
P\left\{\lambda_{1}>\lambda\right\} & =P\left\{\tilde{\lambda}_{1}>\ln z+o(1)\right\}=P\left\{\tau_{1}(-\infty)>\mathscr{L}\right\} \\
& =P\left\{\tilde{\tau}_{\lambda}>\ln z\right\}=z+o(1) .
\end{aligned}
$$

Putting $z=\exp (x)$ we obtain

$$
P\left\{\tilde{\lambda}_{1}>x\right\} \rightarrow \exp (-\exp (x)), x \in R^{1} .
$$

Differentating the right side of (3.45) we get (3.43), where $k=1$.

To find $\lambda_{k}$ it is necessary to take into account that the transformed phase runs through the real axis $k$ times, as it was mentioned earlier. Since every such transition is independent of previous transitions then

$$
P\left\{\tilde{\lambda}_{k}>x\right\}=P\left\{\xi_{1}+\xi_{2}+\ldots+\xi_{k}>x\right\},
$$


where all $\xi_{i}$ are mutually independent and have limit density

$$
p(x)=\exp (x-\exp (x))
$$

((3.43), where $k=1)$.

Consequently the limit density of $\tilde{\lambda}_{k}$ is a $(k-1)$-times convolution of the density (3.46). From this (3.43) follows.

Notice that Theorem 2 may be written in an equivalent form

$$
\lambda_{k}=\frac{\pi^{2}}{\ln ^{2} \mathscr{L}}\left(1-\frac{2 \zeta_{k}}{\ln \mathscr{L}}\right), \quad k=1,2 \ldots
$$

where $-\zeta_{k}$ have the limit density (3.43).

And now we can formulate the main result of the paper. Namely the following theorem is true.

Theorem 5. The joint limit distribution of $\tilde{\lambda}_{k}, \tilde{\lambda}_{k-1} \ldots \tilde{\lambda}_{1}$ coincides with the distribution of the first $k$ eigenvalues in the "pieces" model under the same normalization.

Let us introduce random variables $\hat{\lambda}_{i}, i=1,2 \ldots k$ similar to $\tilde{\lambda}_{i}, i=1,2 \ldots k$ with the same points of partition in the "pieces" model. Obviously

$$
\tilde{\lambda}_{i} \leqq \hat{\lambda}_{i}, \quad i=1,2 \ldots k
$$

and with $\mathscr{L} \rightarrow+\infty$

$$
\tilde{\lambda}_{i}-\hat{\lambda}_{i} \stackrel{(P)}{\longrightarrow} 0, \quad i=1,2 \ldots k \quad(\text { Theorem } 4) .
$$

This means that the limit distribution of vectors $\left(\tilde{\lambda}_{k}, \tilde{\lambda}_{k-1} \ldots \tilde{\lambda}_{1}\right)$ and $\left(\hat{\lambda}_{k}, \hat{\lambda}_{k-1} \ldots \hat{\lambda}_{1}\right)$ are identical.

\section{References}

1. Pastur, L.A.: The spectra of the random self-adjointness operators. Usp. Mat. Nauk 28, 3-64 (1969), (1973). English transl. in Russian Math. Surv. 28, (1973)

2. Kozlov, S.M.: The averaging of the random operators. Mat. Sb. 109 (151) 188-202 (1979)

3. Lipšic, I.M., Gredeskul, S.A., Pastur, L.A.: An introduction to disordered system theory. Moskva : Nauka 1982

4. Molčanov, S.A.: On the nature of the eigenfunctions of one-dimensional disordered structures. Izv. Akad. Nauk SSSR 42, 70-103 (1978); English transl. in Math. SSSR Izv. 12, (1978)

5. Molčanov, S.A.: The local structure of the spectrum of the Random one-dimensional Schrödinger operator. Commun. Math. Phys. 78, 429-446 (1981)

6. Malkin, V.M.: The distribution of the distances between the energetic levels of the particle in one-dimensional random field. Preprint 81-49, IIAF SO Akad. Nauk SSSR, Novosibirsk, 1981

7. Casati, G., Valz-Gris, F., Guarniery, I.: On the connection between quantization of nonintegrable systems and statistical theory of spectra. Let. Nuovo Cimento 28, 279 (1980)

8. Gordin, M.I., Lifšic, B.A.: The central limit theorem for Markov stationary processes. Dokl. Akad. Nauk SSSR 239, 766-767 (1978)

9. Ibragimov, J.A., Linnik, Ju.V.: Independent and stationary connected variables. Moskva: Nauka 1965 
10. Billingsley, P.: Convergence of probability measures. New York: Wiley 1968

11. Narimanjan, S.M.: The central limit theorem for random walk on the circle. Dokl. Akad. Nauk Arm. SSSR 64, 129-136 (1977)

12. Anshelevich, V.V., Vologodskii, A.V.: Laplace operator and Random walk on one-dimensional nonhomogeneous lattice. J. Stat. Phys. 25, 419-430 (1981)

13. Anshelevich, V.V., Khanin, K.M., Sinai, Ya.G.: Symmetric Random walks in Random environments. Commun. Math. Phys. 85, 449-470 (1982)

14. Friedrichs, K.O. : Perturbation of spectra in Hilbert space. Providence, Rhode Island: Am. Math. Society, 1965

15. Feller, W.: An introduction to probability theory and its applications. New York: Wiley 1966

Communicated by Ya. G. Sinai

Received July 15, 1982; in revised form March 14, 1983 
